- Changes in patterns of tooth loss, and an ageing population, may mean that there will be an increase in the number of partially dentate patients.

- The type and extent of occlusal change may compromise restoration of the unopposed tooth and teeth bounding the extraction site.

- Strategies to prevent undesirable tooth movements may be indicated.

\title{
A study of the incidence of overeruption and occlusal interferences in unopposed posterior teeth
}

\author{
H. L. Craddock ${ }^{1}$ and C. C. Youngson ${ }^{2}$
}

\begin{abstract}
Objectives To determine the incidence and extent of overeruption in unopposed posterior teeth; to determine the incidence of occlusal interferences associated with unopposed posterior teeth; and to detect an association between the extent of overeruption and the presence of occlusal interference.

Design The study was a single centre clinical retrospective analysis. Setting The setting was in the Combined Care Clinic of Leeds Dental Institute. The patients were undergoing routine restorative care by final
\end{abstract} year undergraduate dental students during 2000.

Subjects and methods All patients over 18 years of age with one or more unopposed posterior tooth, were invited to participate. A sample group of 120 subjects were recruited. A clinical occlusal examination was carried out and the presence of initial retruded contact position (RCP) contacts and excursive interferences were recorded. Study models were made and digitally scanned.

Outcome measures Measurements of overeruption from the curve of Spee were made from the images obtained, by a single observer. Reliability of the measurements was assessed by means of a Bland Altman Plot. The presence and site of RCP contacts and excursive interferences were recorded by a single observer. Reliability of the measurements was assessed by means of Kappa scores. The association between overeruption and the presence of occlusal interference was examined using Spearmans Correlation Coefficient.

Results A total of 120 subjects were recruited, with a total of 155 unopposed sites. Eighty-one of the sites belonged to male subjects, 74 to female subjects. The mean age of the group was 50.9 years, Standard deviation 13.9. Eighty-three per cent of sites displayed overeruption (95\% Cl 78-90), ranging from under $0.5 \mathrm{~mm}$ to $5.4 \mathrm{~mm}$. The reliability of the observations was found to be good. A total of $51.6 \%$ (95\% Cl 44-60) of unopposed teeth were involved in RCP contacts or excursive interferences. Thirty-two sites were involved in more than one contact or interference. The intra-examiner agreement was found to be good,

$1^{*}$ Lecturer in Restorative Dentistry, Division of Restorative Dentistry, Leeds Dental Institute, The Worsley Building, Clarendon Way, Leeds LS2 9LU 2Professor of Restorative Dentistry, University of Liverpool Dental Hospital and School, Pembroke Place, Liverpool L3 5PS ${ }^{*}$ Correspondence to: H. L. Craddock

Email:H.L.Craddock@leeds.ac.uk

\section{Refereed Paper}

doi:10.1038/sj.bdj.48110832

Received 20.12.02; Accepted 20.05.03

( ) British Dental Journal 2004; 196: 341-348 using Kappa. The findings of the Spearman's correlation, show that if an association exists between overeruption and occlusal interference, it is weak, and of no statistical significance.

Conclusions Eighty-three per cent of unopposed teeth are likely to overerupt, and the extent of the overeruption may be marked. The incidence and extent of overeruption is of clinical significance, not only in terms of treatment planning to prevent undesirable vertical movement, but also in the restoration of the edentulous space. The reliability of observations made in the study was found to be good, justifying the methodology. Fifty-one point six per cent of unopposed teeth are likely to be involved in RCP contacts or excursive interferences. The incidence is of clinical significance when planning the restoration of unopposed teeth, or the associated edentulous space. Only a weak statistical association was demonstrated between the degree of overeruption and the presence of occlusal interferences.

Patterns of tooth loss are changing throughout the population, with more patients remaining dentate until old age. During this time a number of posterior teeth may be lost, leaving antagonists unopposed. Restorative dentists regularly see what is sometimes a dramatic loss of interocclusal space following an extraction of an opposing tooth.

In their 2000 study Kiliaridis et al. ${ }^{1}$ identified that overeruption greater than $2 \mathrm{~mm}$ occurs in 24\% of unopposed teeth, with 18\% having no demonstrable overeruption at all. This leaves a total group with some overeruption of $82 \%$, which in terms of restoration could have a clinical significance. The study was fairly crude, using hand held models and a ruler to place teeth into one of three categories: no overeruption, less than $2 \mathrm{~mm}$ and greater than $2 \mathrm{~mm}$ overeruption. As there was no measurement of the overeruption beyond $2 \mathrm{~mm}$, it is conceivable that in this $24 \%$ of subjects, some overeruptions may have been extreme.

Smith ${ }^{2}$ looked specifically at the effects of the extraction of the upper second molars on the lower second molar position in patients who had had orthodontic extractions. In this particular situation the vertical movement of the mesial cusp of the lower second molar was influenced by the position of the upper first molar on which it may or may not occlude. The distal cusps were not under this influence. Overeruption of the unopposed teeth was found to be statistically significant, and the problem was mainly confined to the distal 
aspect of the teeth. There is no reason to suppose that this movement does not also occur in unopposed adult sites.

This confirmed Kaplan's ${ }^{3}$ findings that in supraerupted teeth not all of the movement was in a vertical direction and there was often also a horizontal or rotational component. Kaplan deduced that this horizontal component may have been due to guidance by partial tooth contact with an antagonist. The last two studies indicate that even leaving a tooth partially opposed may not prevent undesirable vertical rotations.

The unopposed upper first molar, in both healthy mouths and those with some periodontal pathology present, was studied by Compagnon and Woda. ${ }^{4}$ Their study showed that the majority of overeruption occurred in the early years following opposing tooth loss. In later years loss of periodontal support may be superimposed on the picture. In healthy individuals they noted that the gingival margin remained at its original level on the tooth during this occlusal tooth movement. The study found that after 10 years of remaining unopposed, this periodontal migration reversed and root exposure occurred. This was described by the authors as passive eruption. From these findings it is obvious that the appearance of overeruption may have several components, including periodontal growth, passive eruption and active eruption.

Clinically, overeruption of posterior teeth may often complicate the restoration of an edentulous space with either fixed or removable prostheses. In severe cases, most or all of the interocclusal space may be taken up by the vertical movement of the unopposed tooth (Fig. 1), prohibiting restoration of the space without extraction of the overerupted tooth. The extent of overeruption will determine to what extent the overerupted tooth can be reduced (Fig. 2), or the occlusal plane modified.

This change in tooth position could result in the development of an occlusal interference or retruded contact position contact, which may be of relevance in restoration of the teeth involved. The presence of occlusal interferences in the mouth has been noted to have an incidence of between 75-89\% in Agerberg and Sandstrom's study. ${ }^{5}$ This study's subject group consisted of teenagers and young adults. Unilateral tooth contacts in retruded position were found in 75\% of both subject groups. Non-working side contacts were found in $6-13 \%$ of the teenagers and $9-25 \%$ of the
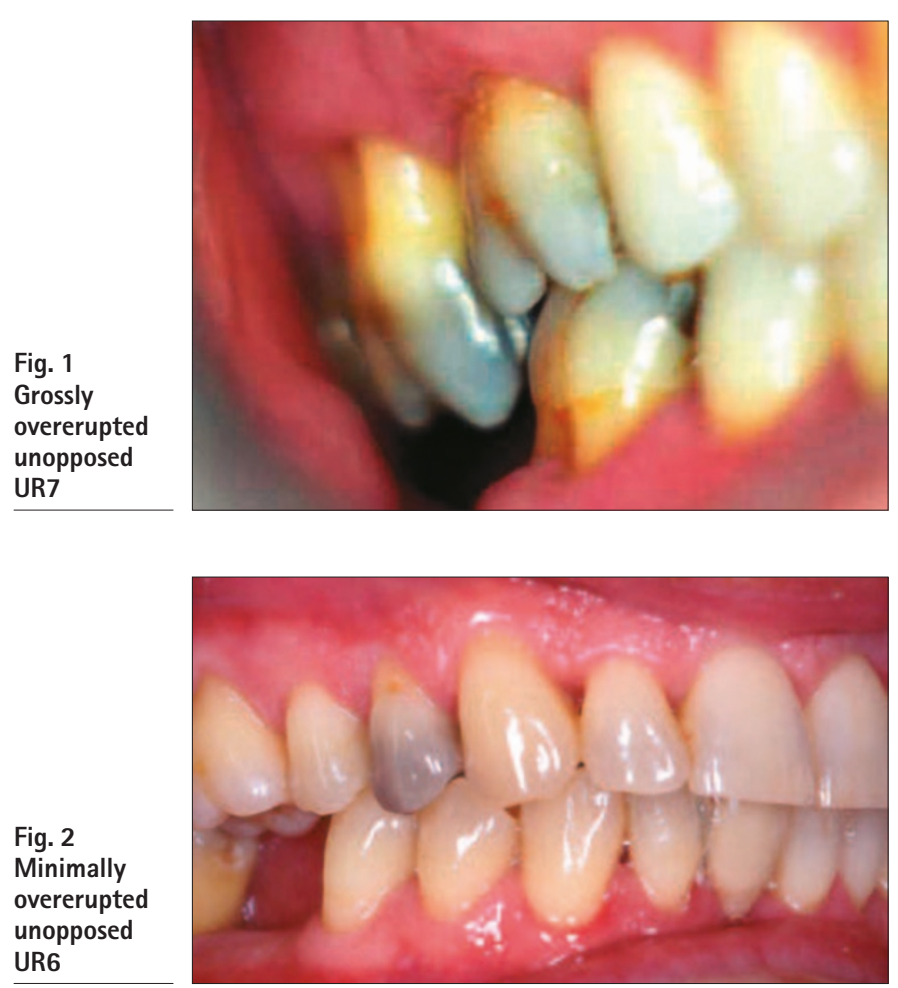

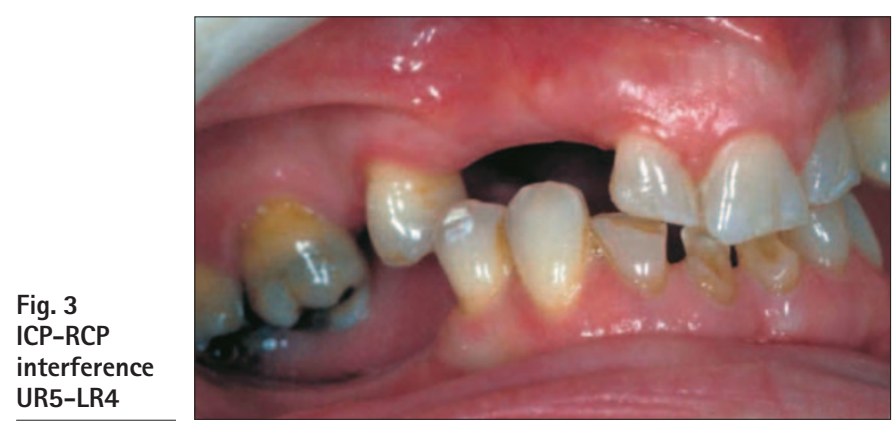

young adults. In total, 89\% of all subjects showed at least one occlusal interference. In older subjects restoration, wear and tooth fracture may change the incidence and distribution of occlusal interferences from those found in the above studies.

The presence of occlusal interferences, whilst not proven to be associated with any pathology, can certainly complicate the restoration of the teeth involved (Fig. 3).

The current investigation will attempt to provide a guide to the extent of overeruption that the clinician is likely to encounter and indicate whether an associated occlusal interference is likely to be present.

\section{AIMS OF THE STUDY}

The purpose of this study is :

- To determine the incidence and extent of overeruption in unopposed posterior teeth.

- To determine the incidence of occlusal interferences associated with unopposed posterior teeth.

- To detect an association between the presence of an occlusal interference and the presence and extent of overeruption of unopposed posterior teeth.

\section{HYPOTHESES}

- No vertical change in tooth position occurs following the loss of an antagonist in posterior teeth.

- Unopposed posterior teeth are not associated with occlusal interferences.

- There is no association between the presence of an occlusal interference and the presence of overeruption in unopposed posterior teeth.

\section{METHODS AND MATERIALS}

\section{Selection criteria}

A total of 120 patients over 18 years of age with one or more unopposed posterior teeth were invited to take part in the study. Patients included in the study had a maximum of two unopposed sites per jaw. Written informed consent was obtained from all subjects following a verbal explanation of the purpose of the study and methods to be used in the investigations. Local ethical committee approval was gained. An explanatory leaflet was provided for each patient. All patients invited to participate agreed to take part.

The patients were recruited from the Combined Care Clinic, which is an area where final year students provide whole patient multi-disciplinary care for regularly attending patients of the institute. No patient had a BPE score in excess of three. It is likely that these patients will be similar to general dental practice patients in their experience of posterior tooth loss.

For the purposes of this study 'posterior' is defined as an upper or lower first or second premolar, or first, second or third molar. We define unopposed as 20\% or less of the occlusal surface in contact with an opposing tooth when examined in intercuspal position. When more than one tooth in the same quadrant was unop- 
posed the tooth nearest to an antagonist was observed. Where this was judged to be equal, both were included in the study.

This group presented with a total of 155 unopposed posterior sites. One hundred and twenty one sites were in the upper arch, 34 sites in the lower arch. Sites belonging to male patients totalled 81, females 74 . The mean age for this group of subjects was 50.9 years, standard deviation 13.94 .

\section{Materials used}

Impressions for study models were made using Kromopan alginate impression material (Lascod, Italy) with tray adhesive: (Fix, De trey, Weybridge, England) in orthodontic stock impression trays (Orthocare, Bradford, UK). Prior to casting, the impressions were disinfected in Perform, (Schulke and Mayer, Germany) and models were subsequently cast in Dentstone KD model stone. (British Gypsum, Newark, England). Trimmed models were scanned using a Black Widow 9636 USB three dimensional scanner (Devcom Ltd, Stirling UK). Analysis of scanned images was carried out using

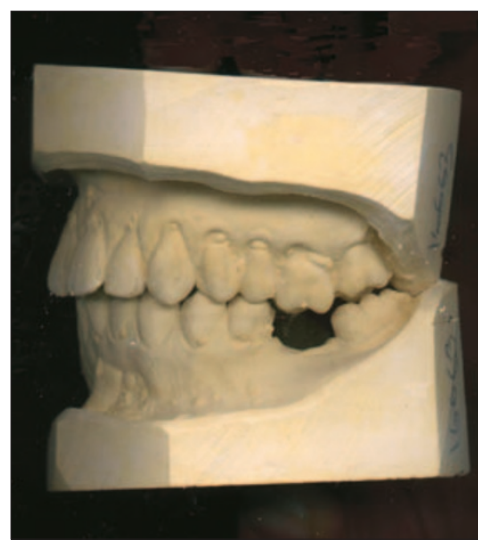

Fig. 4 Scanned image of occluding models
QuickCAD Millenium Edition software (Autodesk Ltd, Surrey, UK).

The models were trimmed in a seven-sided configuration, so that the buccal plane would lie parallel to the scanner surface when the models were placed on it. (Fig. 4)

Initial occlusal examination was made using 8 micron Hanel occlusal marking foils (Roeko, Germany) held in Miller forceps (Ash UK Ltd).

\section{Clinical examination}

The unopposed teeth under investigation were assessed visually for occlusal interferences by one examiner. The presence of occlusal contact or interferences involving unopposed teeth in $\mathrm{RCP}$, protrusion, and on both working and non-working excursions was observed and identified using articulating foils in different colours to identify contacts in different excursions. These markings were removed and the procedure repeated to confirm findings. RCP was reproducibly detected in all subjects.

\section{Re-examination}

Ten per cent of the total sample, every sixteenth subject already examined, was re-examined to determine intra-examiner reliability. The re-examination took place between 3 and 6 months after their initial examination. The intra-examination reliability was then calculated.

\section{Determination of overeruption}

Each study model was scanned with a millimetre scale rule included in each image at a resolution of 1401 DPI at 50\% scale in full colour (Fig. 5). The images were stored in Microsoft 'Picture it' format to retain the threedimensional quality of the image.

The degree of overeruption of the tooth under investigation was measured using QuickCAD software. The degree of overeruption was measured from the curve of Spee to the tip of the

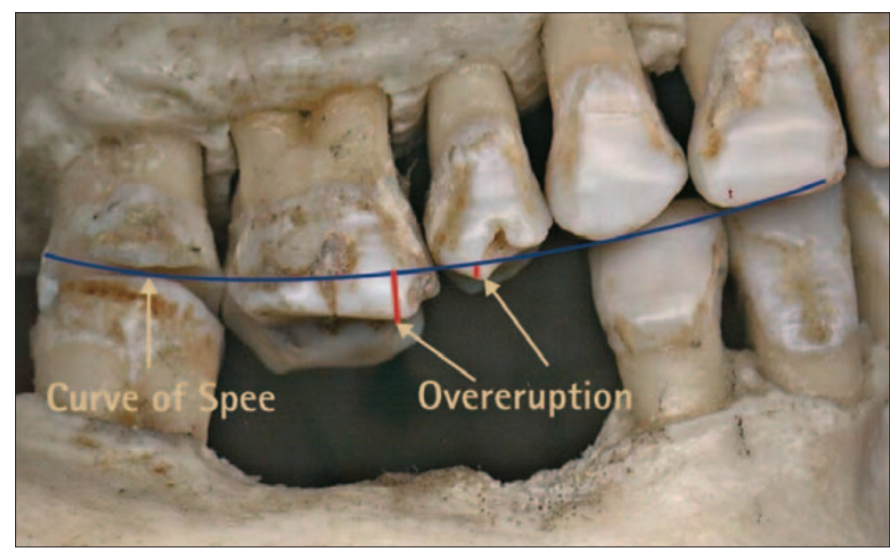

Fig. 6 Overeruption of teeth measured from generated curve of Spee

most occlusally projecting cusp in a direction as closely following the long axis of the tooth as could be determined. Worn and fractured teeth were measured to the most occlusally projecting part of the cusp surface. In many cases the curve was self-evident and could be easily determined from the positions of the existing teeth within that arch. The curve of Spee in Figure 6 is indicated in blue. Measurement of overeruption was from this line to the cusp tips of the two unopposed teeth (shown in red)

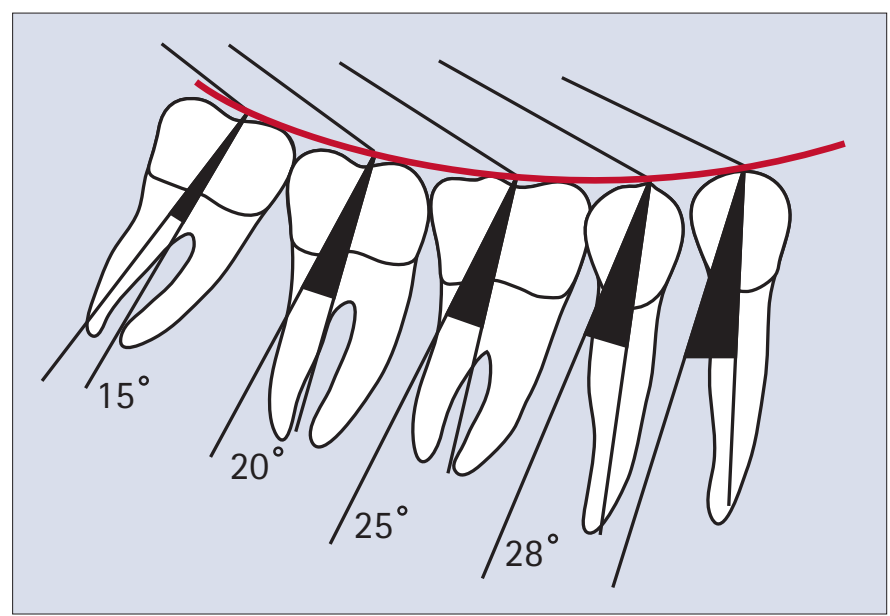

Fig. 7 Posterior tooth progressive differential angle to a tangent drawn on the curve of Spee

In some cases where there was no tooth distal to the unopposed tooth as in the last molar, the curve of Spee needed to be transferred from the opposing arch and superimposed to allow measurement of overeruption to take place. Obviously, in this case, the tooth which would normally oppose the tooth under investigation was missing, and its normal position had to be determined before the curve could be drawn. In order to do this the method described by Orthlieb ${ }^{6}$ was used. This allowed extension of the existing curve, bringing into play the progressive differential angle the posterior teeth display, with respect to a tangent drawn on the curve, demonstrated in Figure 7.

The example shown in Figure 6 cannot have the curve of Spee in the region involving the overerupted third molar drawn directly, as there is no tooth distal to the tooth under examination. We therefore determined the distal portion of the curve using Orthlieb's ${ }^{6}$ method.

In Figure 8, the existing curve of Spee for the lower teeth is drawn in blue. The increase in angulation for the lower third molar was 15 degrees and this was plotted accordingly, shown in green. The final tangent of the curve of Spee could then be drawn, as shown in yellow. Finally the determined curve for this individual was superimposed on the upper arch and from this the degree of overeruption could be measured as described previously (shown in red). 


\section{RESEARCH}
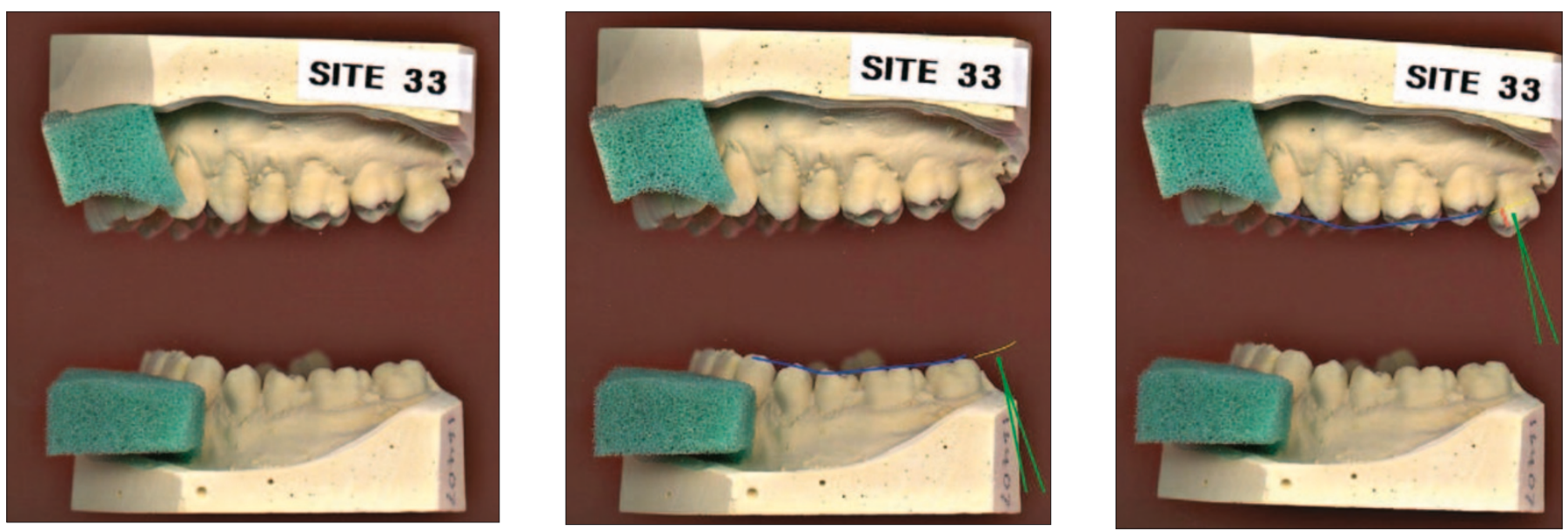

Fig. 8 Measurement of overeruption

Following the correct determination of the curve of Spee for each individual, the measurements of overeruption were recorded. Ten per cent of sites were re-examined to assess intraexaminer reliability, selecting every sixteenth site initially examined.

\section{Statistical analysis}

Before embarking on the study, advice was obtained from a statistician and sample size calculations carried out. Data from previous studies of the incidence of occlusal interferences and overeruption were used to calculate a sample size large enough to allow the results to have a statistical significance.

Standard deviations and confidence intervals were reported for proportional data. A Bland Altman plot ${ }^{7}$ was used to assess and demonstrate the degree of agreement between two samples of overeruption measurements carried out by the same examiner on different occasions.

\section{RESULTS}

\section{Incidence of occlusal interference}

The percentage incidence of occlusal interferences associated with unopposed teeth is shown in Table 1 . The investigation indicated that $48.8 \%$ of upper and $61.8 \%$ of lower, unopposed, sites had associated occlusal interferences (Table 2).

\section{Number and location of occlusal interferences}

Having established the frequency of occurrence of occlusal interferences clinically, it is important to determine whether interferences may be present in more than one excursion. Table 3 demonstrates the number of interferences associated with each unopposed site. It was shown that whilst most sites were associated with an interference in only one excursion, several sites were involved in two interferences and a small number in three. No sites investigated in this study gave rise to interferences in all four excursions. Table 4 shows the number of interferences encountered in each excursion.

\begin{tabular}{lcccc} 
Table 1 Incidence of the presence of occlusal interferences & & \\
\hline $\begin{array}{c}\text { No } \\
\text { interferences }\end{array}$ & $\begin{array}{c}\text { Interferences } \\
\text { present }\end{array}$ & $\begin{array}{c}\text { Percentage with } \\
\text { interference }\end{array}$ & $\begin{array}{c}95 \% \\
\text { confidence } \\
\text { interval }\end{array}$ \\
\hline Number of patients & 75 & 80 & 51.6 & $44-60$
\end{tabular}

\begin{tabular}{lcccc}
\multicolumn{4}{l}{ Table $\mathbf{2}$ Values of upper and lower interferences } \\
$\begin{array}{l}\text { Site under } \\
\text { investigation }\end{array}$ & $\begin{array}{c}\text { No } \\
\text { interferences }\end{array}$ & $\begin{array}{c}\text { Interferences } \\
\text { present }\end{array}$ & $\begin{array}{c}\text { Percentage of sites with } \\
\text { interference }\end{array}$ & $\begin{array}{c}95 \% \\
\text { confidence } \\
\text { interval }\end{array}$ \\
\hline Upper sites & 62 & 59 & 48.8 & $39.9-57.7$ \\
\hline Lower sites & 13 & 21 & 61.8 & $45.4-78.1$
\end{tabular}

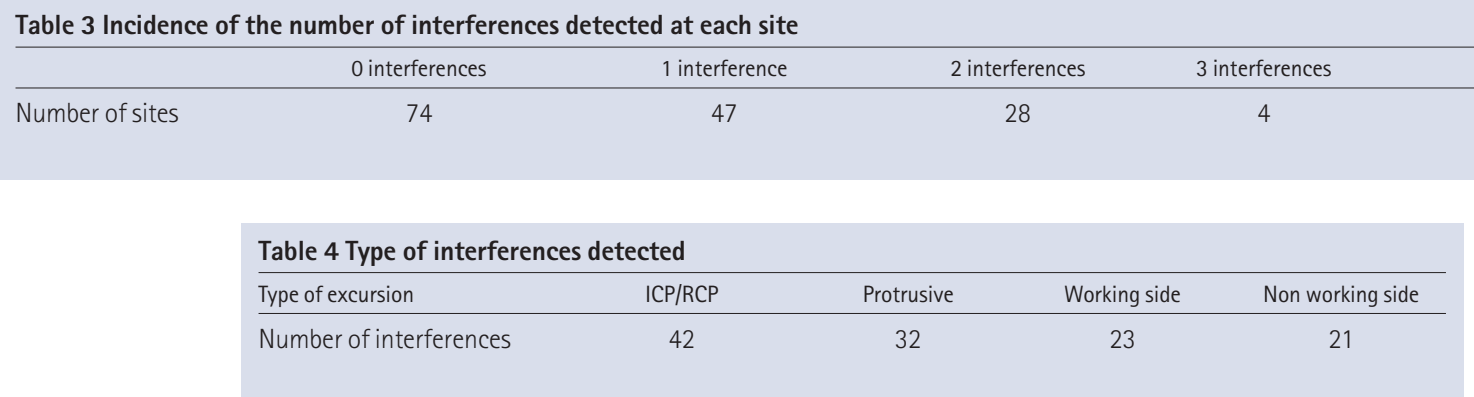




\section{Assessment of clinical examiner reliability - presence of interferences}

Table 5 demonstrates the level of agreement for the same clinical examiner at the two separate examination times. The table demonstrates Kappa scores for each of the groups of interferences ie ICP/RCP, protrusive, working side and non-working side.

\section{Incidence of overeruption}

The presence of overeruption is defined as a measurement in excess of zero from the cusp tip to the curve of Spee. The incidence of overeruption detected in this study is detailed in Table 6.

\section{Measurement of overeruption}

Overeruption measurements are reported in millimeters. The range of overeruption was 0 to $5.39 \mathrm{~mm}$. To enable easier comparison of the degrees of overeruption the data were sorted into groups of $0.5 \mathrm{~mm}$ and this is shown in Table 7. The data are also represented graphically in Figure 9.

\section{Assessment of intra-examiner reliability - measurement of overeruption}

The intra-examiner agreement was determined by drawing a Bland-Altman plot. From this it can be seen that all of the measurements fall within the 95\% confidence interval bands as shown in Figure 10.

\begin{tabular}{lccc}
\multicolumn{2}{l}{ Table $\mathbf{5}$ Intra-examiner agreement } \\
\hline Type of interference & $\begin{array}{c}\text { Kappa } \\
\text { score }\end{array}$ & $\begin{array}{c}95 \% \text { confidence } \\
\text { interval }\end{array}$ & $\begin{array}{c}\text { Strength of } \\
\text { agreement }\end{array}$ \\
\hline ICP/RCP & 0.87 & $0.6-1.2$ & Very good \\
\hline Protrusive & 0.70 & $0.1-1.3$ & Good \\
\hline Working side & 0.56 & $0.1-1.0$ & Moderate \\
\hline Non-working side & 0.63 & $0.1-1.3$ & Good
\end{tabular}

\begin{tabular}{lcccc}
\multicolumn{4}{l}{ Table 6 Incidence of overeruption } \\
$\begin{array}{l}\text { No } \\
\text { overeruption }\end{array}$ & Overeruption & Total & $\begin{array}{c}\text { Percentage with } \\
\text { overeruption }\end{array}$ & $\begin{array}{c}95 \% \text { confidence } \\
\text { interval }\end{array}$ \\
\hline 25 & 130 & 155 & 83.9 & $78-90$
\end{tabular}

Table 7 Measurement of overeruption

\begin{tabular}{lccccccccccccccc}
\hline Overeruption $(\mathrm{mm})$ & 0 & $0.1-0.5$ & $0.51-1.0$ & $1.01-1.5$ & $1.51-2.0$ & $2.01-2.5$ & $2.51-3.0$ & $3.01-3.5$ & $3.51-4.0$ & $4.01-4.5$ & $4.51-5.0$ & $5.01-5.5$ \\
\hline Number of patients & 26 & 4 & 14 & 29 & 29 & 24 & 14 & 3 & 3 & 2 & 1 & 1 &
\end{tabular}

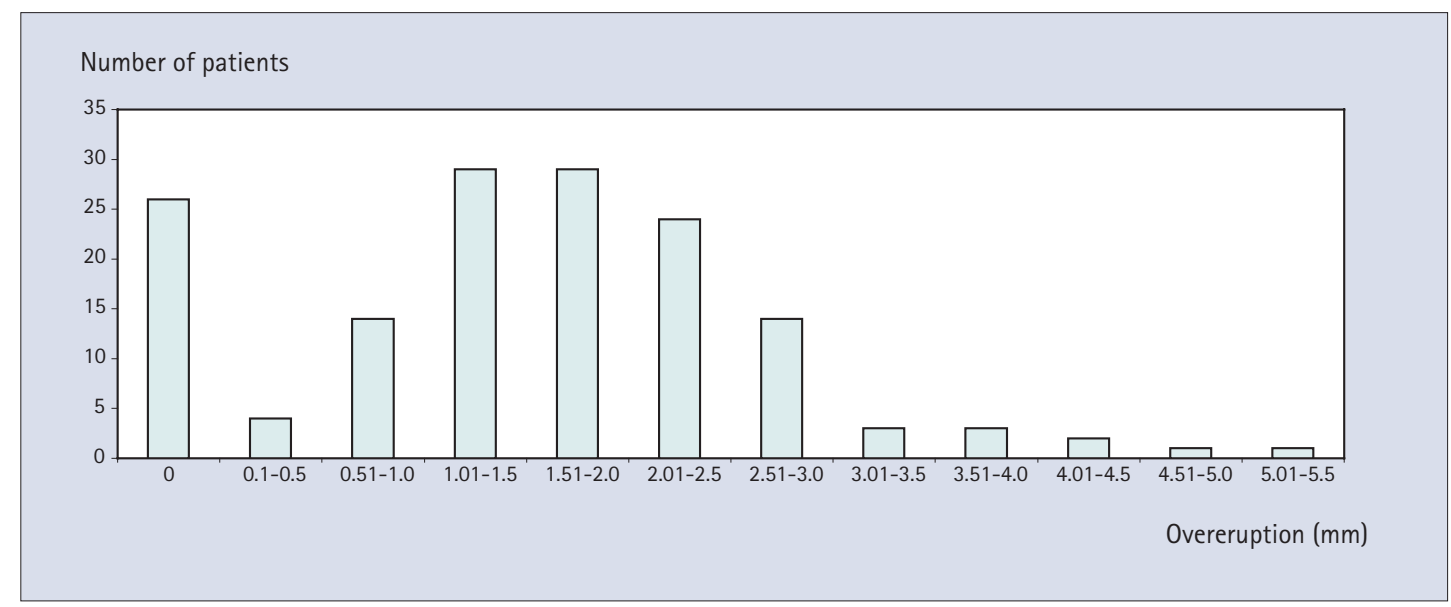

Fig. 9 Degree of overeruption

Fig. 10 Bland Altman plot demonstating intra-examiner agreement

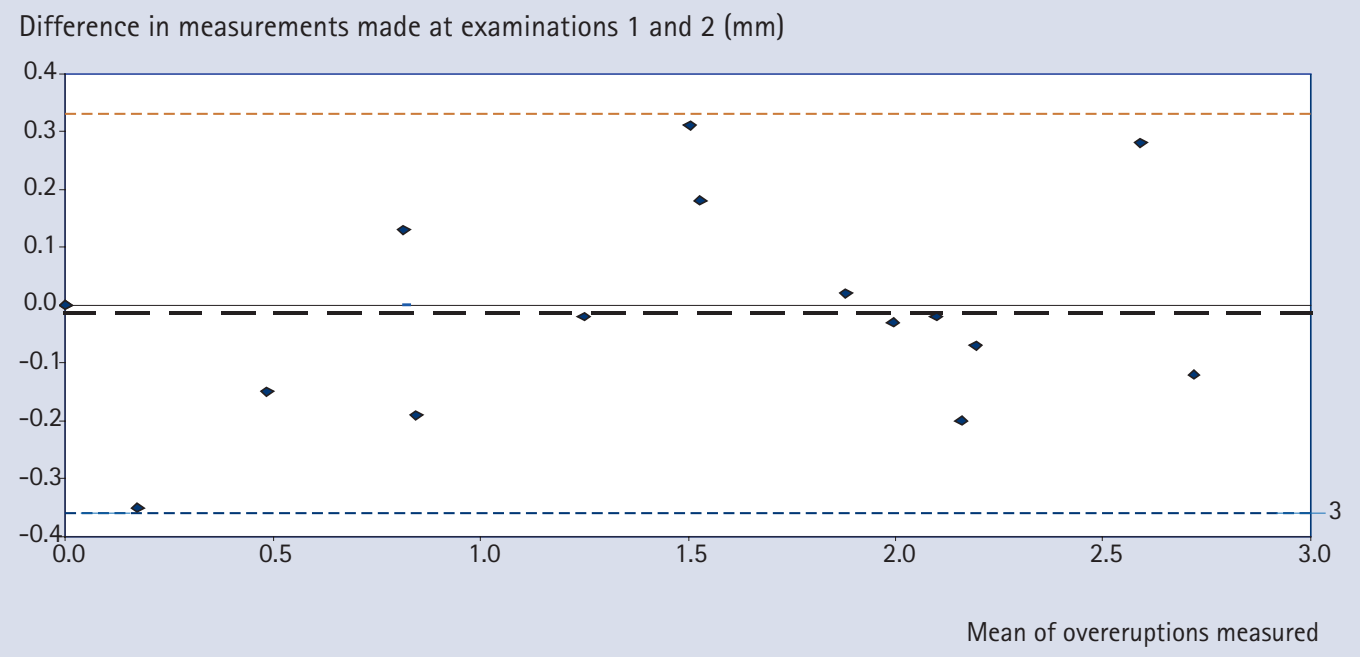




\section{DISCUSSION}

\section{Relevance to restorative dentistry}

Unopposed posterior teeth, in themselves, may pose no difficulty to the patient. However, when restoration of the associated edentulous space becomes necessary, difficulties may arise if the unopposed tooth had been subject to movement from its original point on the occlusal plane. The use of fixed and removable prostheses in the replacement of posterior teeth has, until recently, been fairly low priority for many of our dental patients. Population changes, and the decrease in tooth loss, mean that many patients will present fairly late in life, with one or more posterior edentulous spaces. Patients are now less likely to accept a loss in function and aesthetics, and may generate an increase in the demand for posterior tooth replacement.

Some tooth movement may have occurred since extraction, and this may influence the need for replacement and its complexity. New techniques such as adhesive techniques and dental implants have become available over the last quarter of a century, and our well-informed patient-base is often aware of these possibilities. As with all restorative techniques, adhesive and implant retained restorations are affected by occlusal discrepancies. Knowledge of the role of post extraction tooth movement in the development of occlusal disharmony will be of use when planning these types of restorations.

Most fixed and prosthodontic appliances rely on good occlusal relationships for optimum function and success, preventing occlusal trauma to the abutment teeth and preventing wear and fracture of restorative materials. Even the most successful adhesive materials can debond if occlusal forces are unfavourable, particularly towards the back of the mouth where these are greater.

It is hoped that the information obtained from this study will go some way to increase current knowledge of what occurs to unopposed teeth following loss of function, and form the basis for prosthodontic decision-making, founded on evidence.

\section{Study design}

Detection and measurement of overeruption

It would have been unrealistic and 'self selecting' if the study had been confined to only those models on which the curve of Spee could be seen and drawn directly. The desire to include those patients who had a segment of the curve missing presented several technical challenges and raised many questions:

- Is there a constant curve that can be used for all individuals?

- Is it the same on the right and left sides of the mouth?

- Does it form a constant arc that can be extrapolated?

- If the answers to the first three questions are no, is there any way of determining a relatively accurate curve for a given individual?

- If we can determine the curve it will be dependent on tooth dimension, can this be estimated with any degree of accuracy?

- Is there any readily available technology to assist with this complex process?

As already stated, the distance in an occlusal direction from the curve of Spee is the most accurate way to determine how far a tooth has moved vertically from its original functional position. Orthlieb ${ }^{6}$ described a relatively complex concept, which when accurately applied would allow a curve to be estimated for that side of a given individual. Orthlieb's ${ }^{6}$ work was carried out using orthopantomographs taken prior to the start of orthodontic treatment. These radiographs were most likely taken in response to diagnostic need and not just for the purposes of research. In the case of our study the taking of orthopantomographs purely for the purposes of clinical research could not be ethically justified, as the patient would therefore undergo an

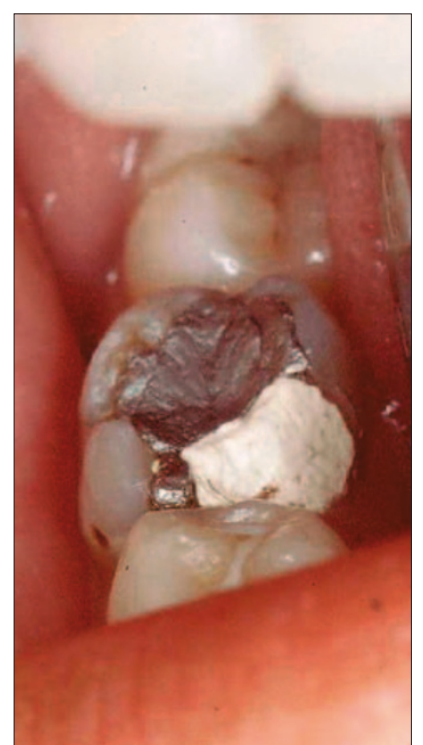
fracture, resulting from a NWSI on a heavily restored tooth
Fig. 11 LR6 mesiolingual cusp

unnecessary radiographic exposure. Also there is degree of enlargement of the dental structures on this type of radiograph, making it unsuitable for measurement of overeruption. It would be difficult technically to transfer the curve drawn on the radiograph to scanned models at the same degree of magnification. The use of study models as the basis of determining overeruption was the most practical, ethically justifiable means of transferring the patients' dentition to a medium for accurate measurement.

Other studies $\left(\right.$ Schirmer $\left.^{8}\right)$ using study models for computer analysis found that the use of a two-dimensional scanner did not allow accurate image focus of tooth structures distant from the scanner surface. It was also concluded that two-dimensional photocopies of scanned images flatten the image and should not be used.

By using a three-dimensional flat bed scanner it was possible to directly import good quality three-dimensional images to the measurement software, together with a simultaneously scanned scale to remove this source of inaccuracy. This is a novel approach to measurement, which may have varied applications.

\section{Detection of occlusal interferences}

The ease with which a patient may achieve the required mandibular positions undertaken during the occlusal examination is very variable, and inevitably some discrepancy is possible. To determine to what extent this may have occurred, a further examination of $10 \%$ of the patients three to six months after the original examination was necessary to determine examiner reliability. There were no patients who had to be excluded from the study because of non-compliance with the examination.

Vallon et al. ${ }^{9}$ found that intra-observer agreement for nonworking side interferences was better than inter-observer agreement, and little difference in consistency was detected for ICPRCP discrepancy. Carlsson et al. ${ }^{10}$ also found similar agreement and recommended that in longitudinal studies all measurements should be carried out by the same observer. It was therefore felt to be justified in this case, to restrict our examination to a single observer. In future studies pilot work should ensure that reproducibility is obtained with a single examiner prior to commencing the main study.

\section{Results of the study}

Significance of the results

When discussing the findings of the study, the significance of these results needs to be assessed. This can be discussed in terms of clinical and statistical significance. These are very different concepts.

A finding would normally be described to be of clinical significance if the clinical diagnosis or management of the patient or condition would be altered by these results. A finding may be of statistical significance if the statistical tests show a detectable difference or association between variables, depending on what is being tested. This may or may not have a clinical significance. 


\section{Overeruption}

Analysis of study models

Incidence of overeruption: The incidence of overeruption in unopposed teeth was high, 83.9\%.

Degree of overeruption: As might be expected there was a wide variation amongst the degrees of overeruption measured. The distribution of the degree of overeruption was skewed, with fewer numbers of sites with severely overerupted teeth. This may be caused by factors such as: extraction of a grossly overerupted tooth due to occlusal discomfort or fracture, dentine sensitivity of exposed root surface, caries or direct soft tissue trauma. Minimal vertical changes in tooth position are unlikely to produce these changes.

Obviously those sites that were free from overeruption were significant, but on examination of the general trend, overeruptions of $1-1.5,1.5-2$ and $2-2.5 \mathrm{~mm}$ were the most commonly encountered values. These values are clinically very significant in relation to the amount of tooth reduction needed during restoration, and particularly in view of the fact that complex occlusal adjustment may be needed for occlusal stability.

Some values encountered were extremely high $(5.39 \mathrm{~mm})$ and these certainly could not be easily treated by conventional restorative techniques alone. These findings indicate that the position of unopposed teeth should be regularly monitored as correction of extreme vertical movements may be impractical or impossible. The use of simple preventive techniques to prevent overeruption, such as that described by Solnit, ${ }^{11}$ may be useful in these cases when deciding on the provision of the definitive restoration. Where the site is bounded the use of a temporary prosthesis may be all that is required. In unbounded sites an adhesive cantilever prosthesis such as those described by Jepson and Allen ${ }^{12}$ will also successfully fulfil this preventive role.

The need for treatment of the more severe overeruptions before the commencement of restorative treatment may not only include conventional orthodontic techniques, but the newer Dahl type appliances may well have an important role in this area, particularly as the appliance is localized and does not usually compromise aesthetics - an important consideration for many adults. The orthodontic 'intrusion' of lower incisors using a bite plane is a well accepted, and generally successful, way of reducing an increased overbite. This principle has been adopted in the development of the 'Dahl' appliance in the treatment of tooth wear. This was described by Dahl et al., ${ }^{13}$ where an anterior bite plane was placed between the upper and lower incisors, and they concluded that the interocclusal space gained between the incisors after wearing the appliance, was due not only to incisal intrusion, but also due to the eruption of the posterior segments.

Intra-examiner reliability: The intra-examiner agreement recorded for the measurement of overeruption was good, as demonstrated by the Bland Altman plot, all values lying within the 95\% confidence interval bands, and the mean difference in measurement close to zero. We may be confident from these findings that the observations and measurements showed a good degree of consistency.

\section{Study limitations}

The software used for measuring overeruption was in itself extremely accurate provided care was taken in its use. As the estimation of the curve of Spee was quite a complex task, obviously some errors may have occurred, although this may have become apparent when examiner reliability was assessed. The complexity of the procedure and its time consuming nature was a minor drawback, but the estimation of only bounded areas would severely limit the relevance of the study findings.

The measurements of overeruption were only made in the occlusal direction. Tooth movements in other planes were not investigated and would have had some role in the development of interferences. Tipping, drifting and rotation of these and the opposing teeth were not investigated in this study.

\section{Occlusal interferences}

Agerberg and Sandstrom ${ }^{5}$ studied the presence of occlusal

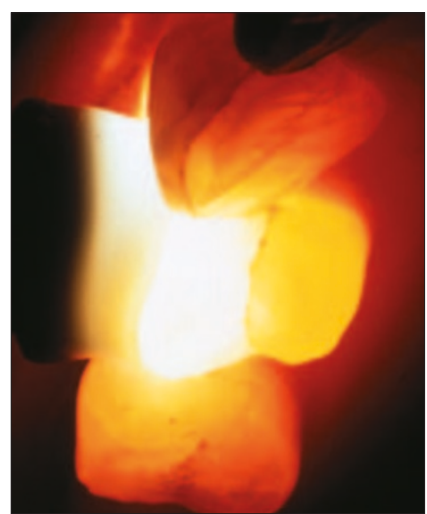

Fig. 12 UR4 mesiodistal longitudinal fracture resulting from a NWSI on an unrestored tooth (courtesy of Primary Dental Care) interferences in the mouth as a whole, and found the incidence to be between $75-89 \%$. This study examined only one occluding site within the mouth and found the incidence to be 51.61\%.

Comparing this with the whole mouth study of Agerberg and Sandstrom, ${ }^{5}$ the incidence in this limited area of study would appear to be high. The high incidence of occlusal interferences involving unopposed teeth is of great clinical significance to the restorative dentist, not only when restoring the unopposed tooth, or replacing the missing opponent. The teeth mesial and distal to the edentulous space may well play a part in occlusal interference and their restoration may be therefore complicated. It was not possible to make a comparison between the incidence of interferences on the upper and lower arches due to the relatively small number of lower unopposed teeth for study.

The most frequently encountered value for interferences was zero. However in those sites displaying an interference, one interference was the most common, followed by two, then three. No sites displayed four interferences. When looking at the location of the interference in the various excursions, ICP/RCP contact (Fig. 3) was the most commonly recorded interference, followed by protrusive interferences.

Clinically the presence of multiple interferences is significant both in occlusal stability, direct occlusal trauma, and the complexity of restoring these and other teeth that may occlude with them. The removal of an initial RCP contact, which was detected in 27\% of the cases with interferences, may allow condylar repositioning after tooth preparation and therefore compromise the occlusal harmony of the finished restoration. The preparation of teeth involved in protrusive interferences, $21 \%$ of the group, will affect the anterior path of guidance, which again needs careful pre-treatment-planning. The presence of a working-side interference will affect the lateral mandibular guidance and again planning of the new occlusal scheme before treatment is essential. Ratcliffe et al. ${ }^{14}$ found statistical association between the presence of lateral excursive interferences and cusp fracture. To confirm these findings further research needs to be carried out into the association between non-working side interferences (NWSI) and cracked cusp syndrome (Figs 11 and 12).

Obviously, when only one examiner is used, there could be concern as to the reliability of the measurements undertaken. The use of the Kappa scores for each type of interference encountered showed in general, good agreement. Kappa was chosen as it allows for any association that may arise by chance and in this case where four different observations are made on the same individual, allows for the non independence of these observations.

It was not possible to determine whether an occlusal interference existed at this site before extraction of the opposing tooth. Statistically, in some of the sites investigated this should be regarded as highly probable. 


\section{CONCLUSIONS}

- There is a high incidence of occlusal interference associated with the presence of unopposed teeth, and the majority of the interferences encountered will be in the retruded contact position and in protrusion.

- There is a high incidence of overeruption of unopposed posterior teeth.

- The incidence and extent of the overeruption is of clinical significance both in terms of treatment planning to prevent overeruption, but also in the restoration of an edentulous space, and pre-treatment re-positioning of the overerupted tooth.

- This study only demonstrated a weak statistical association between the degree of overeruption and the presence of occlusal interferences.

- The reliability of observations made in the study was found to be good, justifying the methodology.

The authors would like to thank Primary Dental Care for permission to use Figure 12.

1. Kiliaridis S, Lyka I, Friede $H_{1}$ Carlsson G E, Ahlqwist M. Vertical position, rotation and tipping of molars without antagonists. Int J Prosthodont 2000; 13: 480-486
2. Smith R. The effects of extracting upper second permanent molars on lower second permanent molar position. Br J Orthod 1996; 23: 109-114.

3. Kaplan P. Drifting, tipping supraeruption and segmental alveolar bone growth J Prosthet Dent 1985; 54: 280-283.

4. Compagnon $D$, Woda A. Supraeruption of the unopposed maxillary first molar. J Prosthet Dent 1991; 66: 29-34

5. Agerberg G, Sandstrom R. Frequency of occlusal interferences: a clinical study in teenagers and young adults. J Prosthet Dent 1988; 59: 212-217.

6. Orthlieb J-D. The Curve of Spee: Understanding the sagittal organisation of mandibular teeth. J Cranio 1997: 15: 333-340.

7. Bland J M, Altman D G. Correlation, regression and repeated data. BrMed J 1994; 30: 896.

8. Schirmer UR, Wiltshire W A. Manual and computer aided space analysis: a comparative study. Am J Orthod Dentofacial Orthop 1997; 112: 676-680.

9. Vallon D, Nilner M, Kopp S. Assessment of occlusal interferences. J Oral Rehabil 1989; 16: $279-286$

10. Carlsson G E, Egermark-Eriksson I, Magnusson T. Intra- and inter-observer variation in functional examination of the masticatory system. Swed Dent J 1980; 4: 187-194.

11. Solnit G S, Aquilino S A, Jordan R D. An etched metal splint to prevent the supereruption of unopposed teeth. J Prosthet Dent 1988; 59: 381-382.

12. Jepson N J A, Allen P F: Short and sticky options in the treatment of the partially dentate patient. BrDent J 1999; 187: 646-652.

13. Dahl BL, Krogstad O, Karlsen K. An alternative treatment in cases with advanced localised attrition. J Oral Rehabil 1975; 2: 209-214.

14. Ratcliff S, Becker I M, Quinn L. Type and incidence of cracks in posterior teeth JProsthet Dent 2001; 86: 168-172. 